\title{
A solution to shape optimization problems using time evolution equations
}

\author{
Daisuke Murai* Atsushi Kawamoto* Tsuguo Kondoh* \\ * Toyota Central Research and Development Laboratories Institute, \\ 41-1, Yokomichi, Nagakute, Aichi, Japan (e-mail: \\ Daisuke-Murai@mosk.tytlabs.co.jp).
}

\begin{abstract}
Keywords: Shape optimization problems, Time evolution equations, Adjoint variable method
\end{abstract}
\section{INTRODUCTION}

This paper deals with a numerical analysis method based on time evolution equations for solving nonparametric boundary shape optimization problems of domain boundaries. Shape optimization problems often appear at the final stage of design.

\section{SHAPE OPTIMIZATION PROBLEM}

Let $\Omega \subset \mathbb{R}^{d}, d=2,3$ be a design domain with boundary $\partial \Omega=\Gamma_{\mathrm{N}} \cup \Gamma_{\mathrm{D}}$ where $\Gamma_{\mathrm{N}}$ is a Neumann boundary and $\Gamma_{\mathrm{D}}$ is a Dirichlet boundary, $D=\cup \Omega$ be a set of design domains. We denote that $\boldsymbol{n}=\left(n_{1}, \cdots, n_{d}\right)$ is an outward normal unit vector on the boundary $\partial \Omega, \mathbf{0}$ is a zero vector and $\boldsymbol{\nabla}=\left(\partial / \partial x_{1}, \cdots, \partial / \partial x_{d}\right)$ for a point $\boldsymbol{x}=\left(x_{1}, \cdots, x_{d}\right)$ in $\Omega$. We define the steady-state heat conduction problem:

$$
\begin{aligned}
& -\boldsymbol{\nabla} \cdot(q \boldsymbol{\nabla} u)=b, \quad \text { in } \Omega, \\
& -(q \boldsymbol{\nabla} u) \cdot \boldsymbol{n}=p, \quad \text { on } \Gamma_{\mathrm{N}}, \\
& u=u_{\mathrm{D}}, \quad \text { on } \Gamma_{\mathrm{D}},
\end{aligned}
$$

where $b \in \mathbb{R}, p \in \mathbb{R}$ and $u_{\mathrm{D}} \in \mathbb{R}$ are given functions, $q>0$ is a given constant. In the problem (1), $b$ presents a heat source in $\Omega, p$ presents a heat flux on $\Gamma_{\mathrm{N}}, u_{\mathrm{D}}$ presents a temperature defined on the boundary $\Gamma_{\mathrm{D}}$ and $q$ presents a thermal conductivity. Using the solution $u$ to the problem (1), we define the objective function as a thermal resistance presented by

$$
f_{0}(\Omega)=\int_{\Omega} b u \mathrm{~d} \boldsymbol{x}+\int_{\Gamma_{\mathrm{N}}} p u \mathrm{~d} \gamma-\int_{\Gamma_{\mathrm{D}}} u_{\mathrm{D}}(q \nabla u) \cdot \boldsymbol{n} \mathrm{d} \gamma
$$

and the constraint function as a volume for domain measure such as

$$
f_{1}(\Omega)=\int_{\Omega} 1 \mathrm{~d} \boldsymbol{x}
$$

A shape optimization problem is defined by

$$
\min _{\Omega}\left\{f_{0}(\Omega) ; f_{1}(\Omega) \leq f_{1}\left(\Omega_{0}\right), u \text { is a solution to }(1)\right\}
$$

where $\Omega_{0} \in D$ is a given initial domain with boundary $\partial \Omega_{0}=\Gamma_{\mathrm{M}} \cup \Gamma_{\mathrm{F}}$ and $f_{1}\left(\Omega_{0}\right)$ is the initial value of $f_{1}$ given as

$$
f_{1}\left(\Omega_{0}\right)=\int_{\Omega_{0}} 1 \mathrm{~d} \boldsymbol{x} .
$$

Here $\Gamma_{\mathrm{M}}$ is a moving boundary, i.e., $\Gamma_{\mathrm{M}}$ is deformed in the computational steps of optimization process and $\Gamma_{\mathrm{F}}$ is a fixed boundary, i.e., $\Gamma_{\mathrm{F}}$ is fixed in the computational steps of optimization process.

A Lagrangian function of the problem (4) is given as

$$
\mathscr{L}\left(\Omega, u, v_{0}, v_{1}, \lambda_{1}\right)=\mathscr{L}_{0}\left(\Omega, u, v_{0}\right)+\lambda_{1} \mathscr{L}_{1}\left(\Omega, u, v_{1}\right),
$$

where $\mathscr{L}_{i}\left(\Omega, u, v_{i}\right), i=0,1$ are Lagrangian functions for $f_{i}, i=0,1$, defined as

$$
\mathscr{L}_{i}\left(\Omega, u, v_{i}\right)=f_{i}+\int_{\Omega}\{\boldsymbol{\nabla} \cdot(q \nabla u)+b\} v_{i} \mathrm{~d} \boldsymbol{x} .
$$

respectively. Here $v_{i}, i=0,1$ are the Lagrange multipliers for the problem (1).

\section{SHAPE DERIVATIVE}

In order to solve the problem (4) by gradient based method, the shape gradient for the objective function $f_{0}$ and the constraint function $f_{1}$ with respect to the variation of $\Omega$ are requested. The shape gradient $\boldsymbol{g}_{i}, i=0,1$ can be obtained using the stationary conditions of $\mathscr{L}_{i}, i=0,1$. The shape gradient of $f_{i}, i=0,1$ are represented as

$$
\frac{\mathrm{d} \mathscr{L}_{i}\left(\Omega, u, v_{i}\right)}{\mathrm{d} \Omega}=\frac{\partial \mathscr{L}_{i}}{\partial u} \frac{\partial u}{\partial \Omega}+\frac{\partial \mathscr{L}_{i}}{\partial v_{i}} \frac{\partial v_{i}}{\partial \Omega}+\frac{\partial \mathscr{L}_{i}}{\partial \Omega}
$$

where $\partial u / \partial \Omega=\delta u$ is a variation of $u, \partial v_{i} / \partial \Omega=\delta v_{i}$ are variations of $v_{i}, i=0,1$.

The stationary condition of $\mathscr{L}_{i}, i=0,1$ for all variations $\delta v_{i}$ of $v_{i}, i=0,1$, such that

$$
\frac{\partial \mathscr{L}_{i}}{\partial v_{i}} \frac{\partial v_{i}}{\partial \Omega}=\int_{\Omega}\left\{(\boldsymbol{\nabla} \cdot(q \boldsymbol{\nabla} u)+b) \delta v_{i}\right\} \mathrm{d} \boldsymbol{x}=0, \quad i=0,1
$$

are equivalent to the condition that $u$ is the solution to the problem (1).

The stationary condition of $\mathscr{L}_{i}, i=0,1$ for all variations $\delta u, \delta u=0$ on $\Gamma_{\mathrm{D}}$ of $u$ such that

$$
\begin{aligned}
\frac{\partial \mathscr{L}_{0}}{\partial u} \frac{\partial u}{\partial \Omega} & =\int_{\Omega}\left\{\boldsymbol{\nabla} \cdot\left(q \boldsymbol{\nabla} v_{0}\right)+b\right\} \delta u \mathrm{~d} \boldsymbol{x} \\
& -\int_{\Gamma_{\mathrm{N}}} \delta u\left\{p+\left(q \boldsymbol{\nabla} v_{0}\right) \cdot \boldsymbol{n}\right\} \mathrm{d} \gamma \\
& +\int_{\Gamma_{\mathrm{D}}}\left\{v_{0}-u_{\mathrm{D}}\right\} \delta(q \boldsymbol{\nabla} u) \cdot \boldsymbol{n} \mathrm{d} \gamma=0, \\
\frac{\partial \mathscr{L}_{1}}{\partial u} \frac{\partial u}{\partial \Omega} & =\int_{\Omega}\left\{\boldsymbol{\nabla} \cdot\left(q \boldsymbol{\nabla} v_{1}\right) \delta u\right\} \mathrm{d} \boldsymbol{x} \\
& -\int_{\Gamma_{\mathrm{N}}} \delta u\left(q \boldsymbol{\nabla} v_{1}\right) \cdot \boldsymbol{n} \mathrm{d} \gamma \\
& +\int_{\Gamma_{\mathrm{D}}} v_{1} \delta(q \boldsymbol{\nabla} u) \cdot \boldsymbol{n} \mathrm{d} \gamma=0
\end{aligned}
$$


are equivalent to the condition that $v_{i}, i=0,1$ are the solutions to the following adjoint problems, respectively:

$$
\begin{aligned}
& -\boldsymbol{\nabla} \cdot\left(q \boldsymbol{\nabla} v_{0}\right)=b, \quad \text { in } \Omega, \\
& -\left(q \nabla v_{0}\right) \cdot \boldsymbol{n}=p, \quad \text { on } \Gamma_{\mathrm{N}}, \\
& v_{0}=u_{\mathrm{D}}, \quad \text { on } \Gamma_{\mathrm{D}}, \\
& -\boldsymbol{\nabla} \cdot\left(q \boldsymbol{\nabla} v_{1}\right)=0, \quad \text { in } \Omega, \\
& -\left(q \nabla v_{1}\right) \cdot \boldsymbol{n}=0, \quad \text { on } \Gamma_{\mathrm{N}}, \\
& v_{1}=0, \quad \text { on } \Gamma_{\mathrm{D}} .
\end{aligned}
$$

Here, we fix $u$ and $v_{i}, i=0,1$ with the solutions of problems (1), (8) and (9). By (7), we have the shape gradients for $f_{i}, i=0,1$ :

$$
\begin{aligned}
& \frac{\mathrm{d} \mathscr{L}_{0}}{\mathrm{~d} \Omega}=\frac{\partial \mathscr{L}_{0}}{\partial \Omega}=\{2 b u-(q \nabla u) \cdot \nabla u\} \boldsymbol{n}=g_{0} \boldsymbol{n}, \\
& \frac{\mathrm{d} \mathscr{L}_{1}}{\mathrm{~d} \Omega}=\frac{\partial \mathscr{L}_{1}}{\partial \Omega}=\boldsymbol{n}=g_{1} \boldsymbol{n} .
\end{aligned}
$$

See Azegami (1994).

\section{SOLUTION TO THE PROBLEM (4) USING TIME EVOLUTION EQUATIONS}

In order to obtain the solution to the problem (4), we introduce following time evolution equations:

$$
\begin{aligned}
& \frac{\partial \boldsymbol{v}(t, \boldsymbol{x})}{\partial t}=\boldsymbol{\nabla} \cdot(c \boldsymbol{\nabla} \boldsymbol{v}(t, \boldsymbol{x}))-\alpha \boldsymbol{v}(t, \boldsymbol{x}), \quad \text { in } \Omega_{0} \times[0, T], \\
& \boldsymbol{v}(0, \boldsymbol{x})=\mathbf{0} \text { in } \Omega_{0}, \\
& -(c \boldsymbol{\nabla} \boldsymbol{v}(t, \boldsymbol{x})) \cdot \boldsymbol{n}=-\left(g_{0}(t)+\lambda_{1} g_{1}(t)\right) \boldsymbol{n} \text { on } \Gamma_{\mathrm{M}} \times[0, T], \\
& \boldsymbol{v}(t, \boldsymbol{x})=\mathbf{0} \text { on } \Gamma_{\mathrm{F}} \times[0, T], \\
& \frac{\partial \boldsymbol{\rho}(t, \boldsymbol{x})}{\partial t}=\boldsymbol{v}(t, \boldsymbol{x}) \text { in }[0, T] \times \Omega_{0} .
\end{aligned}
$$

where $c>0, \alpha \geq 0$ and $T>0$ are given constants. We chose sufficiently large $T$ so that

$$
\left|f_{0}(\Omega(T))-f_{0}(\Omega(T-\delta t))\right| /\left|f_{0}(\Omega(T-\delta t))\right| \leq \varepsilon_{0}
$$

holds for a small time step $\delta t$ and given small constant $\varepsilon_{0}>0 . \lambda_{1}$ denotes a Lagrange multiplier for $f_{1}$. In this paper, we set $\lambda_{1}$ as

$$
\lambda_{1}=-\frac{\left\|g_{0}(t) \boldsymbol{n}\right\|}{\left\|g_{1}(t) \boldsymbol{n}\right\|} \exp \left(\beta f_{1}\right), \quad\|\cdot\|=\sqrt{\langle\cdot, \cdot\rangle},
$$

so that KKT condition $g_{0}(T) \boldsymbol{n}+\lambda_{1} g_{1}(T) \boldsymbol{n}=\mathbf{0}, \lambda_{1} f_{1}(T)=$ $0, \lambda_{1} \geq 0, f_{1}(T) \leq 0$ holds at the end of optimization process. Here $\beta>0$ is the given constant for controlling the violation of constraint function during the optimization process (See Kawamoto (2013)). We consider the violation of $f_{1}$ in the optimization process of computation and chose $\beta>0$ so that $f_{1} \leq 0$ holds for all $t \in[0, T]$. The solution to the problem (4) is obtained by $\Omega=\Omega_{0}+\boldsymbol{\rho}$.

\section{NUMERICAL EXAMPLE}

We analyze a two-dimensional problem related to a steadystate heat conduction problem (1). Fig. 1 shows the initial domain $\Omega_{0}$ with boundary $\partial \Omega_{0}=\Gamma_{\mathrm{M}}$ sup $\Gamma_{\mathrm{F}}$. Fig. 2 shows a design domain $\Omega$ with boundary $\partial \Omega=\Gamma_{\mathrm{N}} \cup \Gamma_{\mathrm{D}}$ where $\Gamma_{\mathrm{N}}=\Gamma_{\mathrm{N} 1} \cup \Gamma_{\mathrm{N} 2}$. Table 1 shows problem settings of the problem (10) and (1). Fig. 3 shows the solution $u$ to the problem (1) in the initial domain $\Omega_{0}$ and the mesh used in this analysis. Fig. 4 shows the solution $u$ to the problem (1) in the optimized domain $\Omega$. Fig. 5 shows the history of objective function and constraint function during the optimization process. In fig. 5, objective function and constraint function are normalized by using each initial values.
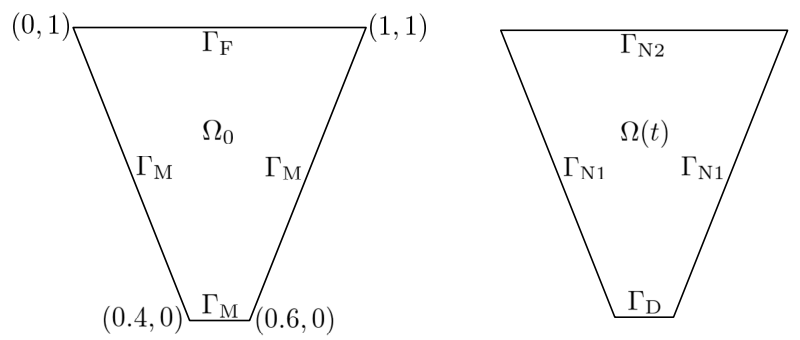

Fig. 1. The original domain $\Omega_{0}$ with boundary $\partial \Omega_{0}$

Fig. 2. A design domain $\Omega$ with boundary $\partial \Omega$

Table 1. Problem settings of the problem (10) and (1)

\begin{tabular}{ll} 
Problem & Settings \\
\hline problem $(10)$ & $T=1.5, c=1, \alpha=0, v_{2}=0$ on $\Gamma_{\mathrm{M}} \times[0, T]$ \\
problem $(1)$ & $q=1, b=0, p=0$ on $\Gamma_{\mathrm{N} 1}, p=-1$ on $\Gamma_{\mathrm{N} 2}, u_{\mathrm{D}}=0$ \\
equation $(12)$ & $\beta=100$
\end{tabular}

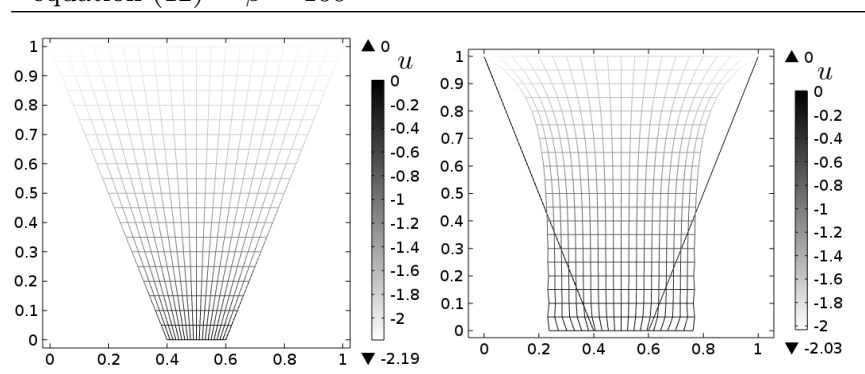

Fig. 3. The solution to the Fig. 4. The solution to the problem (1) in $\Omega_{0}$ problem (1) in $\Omega$

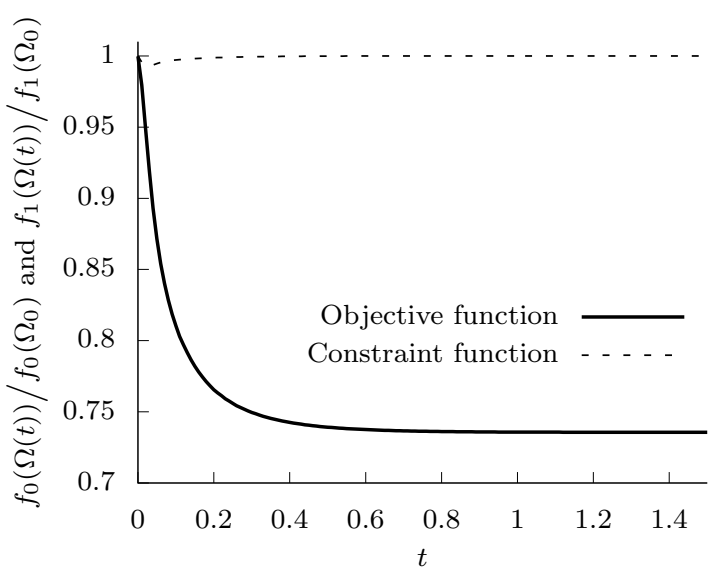

Fig. 5. The history of objective function and constraint function

\section{REFERENCES}

H. Azegami. Solution to domain optimization problems. Trans. Japan Society Mechanics and Engineering 60(574): pages 1479-1994, 1994.

A. Kawamoto, T. Matsumori, T. Nomura, T. Kondoh, S. Yamasaki, S. Nishiwaki. Topology optimization by a time-dependent diffusion equation. International Journal for Numerical Methods in Engineering 93(8): pages 795-817, 2013. 